\title{
Biological Pathways and Gene Networks Link Inflammation and Vascular Remodeling to Both Heart Failure with Preserved and Reduced Ejection Fraction in Women across Ethnicities
}

Qing Liu, Ph.D. ${ }^{1,2}$, Kei Hang K. Chan, Ph.D. ${ }^{2,3}$, Alan R. Morrison, M.D., Ph.D. ${ }^{4,5}$, Stephen T. McGarvey, Ph.D. ${ }^{1}$, Xi Luo, Ph.D. ${ }^{6}$, James G. Wilson, M.D. ${ }^{7}$, Adolfo Correa, M.D., Ph.D. ${ }^{8}$, Alexander P. Reiner, M.D. ${ }^{9,10}$, Jie Li, Ph.D. ${ }^{1,2}, *$ Simin Liu, M.D., Sc.D. ${ }^{1,2,5}$, *Wen-Chih Wu, M.D. ${ }^{1,4,5}$

${ }^{1}$ Department of Epidemiology, School of Public Health, Brown University, Providence, RI 02912, USA

${ }^{2}$ Center for Global Cardiometabolic Health, Brown University, 121 South Main St, Providence, RI, 02903

${ }^{3}$ Department of Biomedical Sciences, City University of Hong Kong, Hong Kong

${ }^{4}$ Providence Veterans Affairs Medical Center, Providence, RI 02908, USA

${ }^{5}$ Alpert Medical School at Brown University, Providence, RI 02903, USA

${ }^{6}$ Department of Biostatistics and Data Science, School of Public Health, University of Texas Health Science Center at Houston, Houston, TX 77030, USA

${ }^{7}$ Department of Physiology and Biophysics, University of Mississippi Medical Center, Jackson, MS 39216

${ }^{8}$ Department of Medicine, University of Mississippi Medical Center, Jackson, MS 39216

${ }^{9}$ Department of Epidemiology, University of Washington, Seattle, WA 98195, USA

${ }^{10}$ Division of Public Health Sciences, Fred Hutchinson Cancer Research Center, Seattle, WA 98109, USA. 


\section{*Co-Corresponding Authors:}

Simin Liu simin_liu@brown.edu

Center for Global Cardiometabolic Health and Department of Epidemiology, Brown

University, 121 South Main St, Providence, RI, 02903

\section{Wen-Chih Wu Wen-Chih.Wu@va.gov}

Center of Innovation in Long Term Services \& Support, Providence VA Medical Center,

Providence, RI

Division of Cardiology, Department of Medicine, Alpert Medical School, Brown University, Providence, RI

\section{Abstract}

Introduction: Heart failure (HF) is understudied among women; especially, genomic evidence implicating shared or unique mechanisms of HF with respect to reduced or preserved ejection fraction (HFrEF, HFpEF) is lacking across ethnic populations of women. Prior genome-wide association studies (GWAS) have identified approximately 30 suggestive genetic variants for HF, although none have been specifically linked to HFrEF or HFpEF.

Objectives: We aimed to define, replicate, and annotate genetic variants to $\mathrm{HFrEF}, \mathrm{HFpEF}$, or both, as well as to investigate potential biological mechanisms underlying HFrEF and HFpEF among African American (AA) and European American (EA) women in three wellcharacterized, high-quality prospective cohorts, the Women's Health Initiative (WHI) study, the Jackson Heart Study (JHS), and the Framingham Heart Study (FHS).

Methods: GWAS analysis on HFrEF and HFpEF were first performed among 7,982 AA and 4,133 EA in the WHI, followed by pathway analysis employing two independent methodological platforms (GSA-SNP and Mergeomics) curating KEGG, Reactome, and 
BioCarta pathway databases. GWAS signals and biological pathways identified using the WHI were replicated in the JHS and FHS. For all replicated pathways, we performed crossphenotype and cross-ethnicity validation analyses to examine shared pathways between HFrEF and HFpEF, and phenotype-specific pathways, across ethnicities. We further prioritized key driver genes for HF according to specific pathways identified.

Results: We validated one previously reported genetic locus and identified six new ones, among which one locus was allocated to HFrEF and five to HFpEF. Additionally, we defined five biological pathways shared between HFrEF and HFpEF and discovered six HFpEFspecific pathways. These pathways overlapped in two main domains for molecular signaling: 1) inflammation and 2) vascular remodeling (including angiogenesis and vascular patterning), involving key driver genes from collagen and HLA gene families.

Conclusions: Our network analysis of three large prospective cohorts of women in the United States defined several novel loci for HF and its subtypes. In particular, several key driver genes reinforce the mechanistic role of inflammation and vascular remodeling in the development of HF, especially HFpEF. Given that therapeutic strategies developed for left ventricular dysfunction have had limited success for HFpEF, several new targets and pathways identified and validated in this study should be further assessed in risk stratification as well as the design of potential new HF interventions. 


\section{Introduction}

According to the American Heart Association (AHA), approximately 6.5 million U.S.

3 adults have heart failure (HF) in $2018^{1}$, representing a major cause of morbidity and mortality

4 in the United States. Heart failure is phenotypically and genetically heterogeneous, and much

5 remains unknown about the etiology for subtypes of HF, including HF with preserved

6 ejection fraction (HFpEF) and HF with reduced ejection fraction (HFrEF). Of note as many

7 as $40-71 \%$ of patients have HFpEF for which limited clinical treatment options are available

8 with little to no impact on outcomes ${ }^{2}$. Moreover, HF is understudied in women, who

9 experience a higher mortality than $\operatorname{men}^{1}$. African American (AA) women have the highest

10 HF incidence, followed by Hispanic Americans (HA) and European Americans (EA), and

11 morbidity and mortality are twice as high for AA women relative to $\mathrm{EA}^{3-6}$. Observational

12 studies have shown that while patients with HFpEF and HFrEF often display similar clinical

13 symptoms, they often have markedly different risk factors, underlying pathophysiological

14 processes, and responses to clinical therapies ${ }^{7}$. Many therapies with unequivocal benefit in

15 HFrEF have failed to show efficacy for $\mathrm{HFpEF}^{8,9}$. Thus, there is a urgent need to further

16 research biological pathways and gene networks for HFpEF and HFrEF that could provide

17 mechanistic insight into the disease processes and identify potential targets for novel

18 treatment modalities, particularly for women, who are disproportionately affected by $\mathrm{HF}^{1}$.

19 Recent candidate gene studies and genome-wide association studies (GWAS) have

20 identified several genetic loci (such as ADRB1, USP3, ITPK1, and BAG3 genes ${ }^{10,11}$

21 associated with HF risk. However, these studies are primarily focused on genes associated

22 with inherited HFrEF and are limited by reproducibility, effect size, and lack of ethnic

23 diversity ${ }^{10,11}$. Although women are at higher risk of HF, no studies to date have

24 examined/observed sex-specific genetic variants. Moreover, few studies have directly

25 investigated the genetic mechanisms underlying the two HF subtypes, HFpEF and 
$26 \mathrm{HFrEF}^{12,13}$; and no studies were conducted in an ethnically diverse population of women. As

27 genes tend to behave conjointly on HF processes, analyzing a cluster of genes with related

28 biological functions, using an integrative pathway and network approach ${ }^{14,15}$, improves the

29 statistical power to identify genetic variants of biological importance. To enhance the

30 understanding of biological mechanisms underlying different HF phenotypes (HFpEF versus

$31 \mathrm{HFrEF}$ ) as well as genomic and ethnic diversity in women with HF, we therefore investigated

32 genetic risk factors and biological pathways predisposing to $\mathrm{HF}$ and its subtypes among AA

33 and EA women in the Women's Health Initiative (WHI) study. We replicated our findings

34 using AA women from the JHS, EA from FHS, and HA women from the WHI.

Methods

37 Study Population

The WHI study enrolled 161,808 postmenopausal women aged between 50 and 79

years old from 1993 to 1998 . The original WHI study has two major components: a partial

41 factorial randomized clinical trial (CT) including 68,132 participants and an observational

42 study (OS) of 93,676 participants. The detailed study design has been reported elsewhere ${ }^{16}$.

43 Briefly, medical records from enrollment through September 2014 for 44,174 WHI

44 participants, including all women randomized to the hormone trial component $(n=27,347)$

45 and all AA participants $(n=11,880)$ and HA participants $(n=4,947)$ from the CT and the

46 OS, were sent to the University of North Carolina (UNC) for HF adjudications.

47 Of the participants enrolled in the WHI-OS, 8,515 self-identified AA women had

48 consented to and were eligible for the WHI-SNP Health Association Resource (SHARe), and

49 of the participants enrolled in the WHI hormone trial, 4,909 EA women were included in the

50 WHI-Genomics and Randomized Trials Network (GARNET). After quality control, the 
51 standard GWAS and pathway analyses were conducted among 8,298 AA and 4,257 EA

52 participants of the WHI. Considering the lack of replication cohort of HA participants in the

53 WHI-SHARe, we treated the HA participants as a replication sample in the pathway analysis.

\section{Population for validation and replication}

56 The current study included three populations as validation and replication: AA in the

57 Jackson Heart Study (JHS), EA in the Framingham Heart Study (FHS), and HA in the WHI-

58 SHARe. Considering the WHI only enrolled postmenopausal women, we replicated the

59 proposed analyses among women in the JHS and FHS. The main FHS enrolled three

60 generations: the original generation (started in 1948), offspring generation (started in 1971),

61 and generation three (started in 2005). Because of the poor measurement of left ventricular

62 ejection fraction (LVEF) among the original generation and the relatively young age of

63 generation three (baseline age $<40$ years old), we only included the offspring generation in

64 the analysis. In total, we conducted the proposed analyses among 1,871 AA women in the

65 JHS, 1,764 EA women in the FHS, and 3,461 HA women in the WHI-SHARe.

Definition of Heart Failure

In the WHI, HF adjudication was based on the Atherosclerosis Risk in Communities

69 (ARIC) classification guidelines ${ }^{17}$, in which HF was defined as having acute decompensated

$70 \mathrm{HF}(\mathrm{ADHF})$ and chronic stable $\mathrm{HF}^{18}$. Participants with adjudicated HF were further classified

71 as HFrEF or HFpEF according to their LVEF. For patients with ADHF, those with LVEF <

$7245 \%$ were considered as HFrEF and those with LVEF $\geq 45 \%$ were considered as HFpEF. For

73 patients with chronic stable HF, baseline LVEF or lowest estimated LVEF on medical

74 records were used to classify HF subtypes. Similar criteria were applied to replication 
75 cohorts, the JHS and FHS. Participants without LVEF were excluded from the analysis

$76 \quad(n=440)$.

77

Genotype Data

Genome-wide genotyping of the WHI-SHARe and JHS participants were performed

using the Affymetrix 6.0 array (Affymetrix, Inc, Santa Clara, CA), and WHI-GARNET and

81 FHS participants were genotyped using Illumina HumanOmni1-Quad SNP platform

82 (Illumina, Inc, San Diego, CA). As the gene chips used for genotyping are designed to

83 capture common genetic variants, genetic variants with frequency $\geq 5 \%$ were genotyped.

84 Reference panels from the 1000 Genomes (1000G) Project Consortium (Version 3, March

852012 release), which provide near complete coverage of common genetic variation with

86 minor allele frequency $\geq 0.5 \%$, were used for genotype imputation.

$88 \quad \underline{\text { Statistical Analysis }}$

Genome-wide association analyses

90 We performed standard GWAS analysis for HFrEF and HFpEF for AA and EA

91 women, using multivariable logistic regressions. The regression models were implemented

92 using allelic dosage at each SNP (single-nucleotide polymorphism) as the independent

93 variable, with covariate adjustment for age, $\operatorname{age}^{2}$, and first four principal components (PCs)

94 for global ancestry in all three cohorts. We also adjusted for region in two WHI cohorts, and

95 randomized hormone treatment group and baseline hysterectomy status in the WHI-

96 GARNET study. Since the associations between germline genetic variants and HF are not

97 confounded by demographic and lifestyle factors, no other confounders were adjusted in the

98 GWAS analysis. The general form of the GWAS model is specified as follows:

$$
\text { logit } \operatorname{Pr}[Y \mid G, V]=\alpha_{0}+\alpha_{g} G+\alpha_{v} V \text {, }
$$


100 where Y denotes HF subtype, G denotes SNPs, and V denotes adjusted covariates. Common

101 genetic variants reaching the suggestive significance $\left(5 \times 10^{-6}\right)$ were identified as potential

102 GWAS hits. Suggestive SNPs were validated in the JHS and FHS using nominal $P$ value <

1030.05 , followed by cross-ethnicity meta-analyses combining AA, EA, and HA women from

104 WHI, JHS, and FHS using METAL ${ }^{19}$ (FDR-adjusted q value < 0.05).

106 Pathway analysis

107 We obtained knowledge-driven metabolic and signaling pathways from three

108 databases: the Kyoto Encyclopedia of Genes and Genomes $(\mathrm{KEGG})^{20}$, Reactome $^{21}$, and

109 BioCarta $^{22}$. SNPs showing potential associations with HF subtypes ( $P$ value $<0.05$ in GWAS)

110 were mapped to relevant genes based on their chromosome locations or functions, and further

111 mapped to biological pathways. Each pathway was tested for enrichment of genetic signals

112 for HFrEF and HFpEF by ethnic groups. To avoid potential biases due to a particular method,

113 we applied two different well-established methods based on known biological pathways: 1)

114 GSA-SNP ${ }^{14}$, and 2) Mergeomics ${ }^{15}$. Pathways were defined as significant if they met the

115 following criteria: 1) identified by both methods from the WHI study with a FDR-adjusted q

116 value $<0.2$; and 2) validated by GSA-SNP or Mergeomics with a significant $P$ value after

117 Bonferroni correction in JHS (as replication of WHI-AA) and FHS (as replication of WHI-

118 EA). We then performed cross-phenotype and cross-ethnicity analyses in WHI to examine

119 shared pathways between HFrEF and HFpEF, as well as phenotype-specific pathways, across

120 ethnicities (AA, EA, and HA).

122 Key Driver Analysis for Identification of Key Regulatory Genes for HF-related Pathways

123 As hundreds of genes are involved in the biological pathways, we seek to further

124 prioritize key driver $(\mathrm{KD})$ genes, defined as genes that played a central role in the disease 
125 progress and once perturbed, should have major impact on many other genes. We integrated

126 all genes involved in significant pathways with seven Bayesian networks and one protein-

127 protein interaction network using KD analysis methods ${ }^{15,23,24}$. We designed a normalized

128 rank score (NRS) to summarize the consistency and strength of identified KD genes across

129 multiple networks ${ }^{25}$, where $N R S=\frac{C_{K D}}{N} \times \sum_{i=1}^{C_{K D}} R_{K D i} ; C_{K D}$ is the count of networks from

130 which a KD was identified; $C_{K D}$ is then normalized by total number of networks $\mathrm{N}$ to

131 represent the consistency of a KD across all networks tested (Bayesian networks from seven

132 tissues, including adipose, blood, brain, islet, liver, kidney, and muscle, and one protein-

133 protein interaction). The KD strength is represented by the summation of normalized

134 statistical rank in each network $i\left(R_{K D i}\right)$ across all networks from which the KD is identified;

$135 R_{K D i}=\frac{R a n k_{K D i}}{N_{K D i}}$, which was calculated by dividing the rank of a KD based on the $P$ values of

136 the Fisher exact test in descending order $\left(\operatorname{Rank}_{K D i}\right)$ by the total number of KDs identified

137 from a network $i\left(N_{K D i}\right)$. KDs with high NRS were those with high network enrichment for

138 pathways and high consistency across tested networks.

$140 \quad$ Results

141 Among WHI participants, 860 (10.4\%) AA, 601 (14.1\%) EA, and 165 (4.7\%) HA

142 were initially identified as having HF. After excluding those without LVEF measurement

143 (316 WHI-AA and 124 WHI-EA), we performed primary analyses among 7,982 AA and

1444,133 EA women in the WHI, and replication analyses among 1,853 AA women in the JHS,

1451,755 EA women in the FHS, and 3,461 HA women in the WHI. The descriptive statistics on

146 demographic and lifestyle factors of each study population are shown in Table 1. Compared

147 to WHI-EA, WHI-AA women were younger in age and less physically active, had higher

148 BMI and lower intakes of alcohol and total calories, and with a higher proportion of

149 cardiovascular disease and diabetes. 
151 Identification of Significant Genetic Loci Using Standard GWAS Analysis

152 In the validation analysis of previously reported 30 loci for HF from the GWAS

153 catalog $^{26}$, we validated one locus and further allocated it to HFpEF in the WHI, JHS, and

154 FHS populations with FDR-adjusted q value $<0.05$. The validated SNP rs4420638 is located

155 on chromosome 19 and close to $A P O E$ and $A P O C$ genes. Detailed information regarding the

156 validated locus can be found in Supplemental Table 1.

157 The standard GWAS results for HFrEF and HFpEF within WHI-AA $(n=7,982)$ and

158 WHI-EA $(n=4,133)$ are shown in the Manhattan plots (Supplementary Figure 1). Among

159 AA, this discovery analysis revealed one significant $\left(P<5 \times 10^{-8}\right.$, rs 35900865$)$ and 57

160 suggestive $\left(P<5 \times 10^{-6}\right)$ SNPs related to HFrEF, and three significant $\left(P<5 \times 10^{-8}\right.$, rs 7834398 ,

161 rs78668964, and rs12203350) and 94 suggestive $\left(P<5 \times 10^{-6}\right)$ SNPs related to HFpEF.

162 Among EA, we failed to identify significant SNPs, but found 50 suggestive $\left(P<5 \times 10^{-6}\right)$

163 SNPs related to HFrEF and 47 SNPs for HFpEF.

164 In the replication analysis for AA women among JHS $(n=1,853)$ participants, eight

165 SNPs from four loci (lead SNPs: rs12067046, rs 114553497, rs10229703, and rs149663839)

166 out of 94 SNPs for HFpEF reached the threshold of $P<0.05$. In the replication analysis for

167 EA women among FHS (n=1,755) participants, one SNP (rs 12719020) reached the $P<0.05$

168 threshold among the 50 suggestive SNPs for HFrEF; and 19 SNPs (concentrated on

169 chromosome 16, lead SNP: rs 12599260) among the suggestive 47 SNPs for HFpEF reached

170 the threshold of $P<0.05$. The effect of all lead SNPs on HF was in the same direction in the

171 discovery population and the ethnicity-specific replication population. In the cross-ethnicity

172 meta-analysis combining AA, EA, and HA women from the WHI, JHS and FHS, all loci

173 passed ethnicity-specific validation and were further validated with FDR-adjust q value of < 
1740.05 (Table 2). More information regarding the newly discovered loci can be found in

\section{Supplemental Table 1.}

177 Identification of Biological Pathways Using Integrative Pathway Analysis

178 We initially identified 21 pathways for HFrEF (nine for EA and 12 for AA) and 42

179 pathways for HFpEF (31 for EA and 17 for AA) among WHI participants, of which 11

180 pathways were validated for HFrEF and 15 pathways for HFpEF, among the JHS and FHS

181 women. The results of cross-phenotype and cross-ethnicity analysis were presented in Table

1823 and Supplemental Tables 2 and 3. Based on the functions of the pathways, we identified

183 two main overarching domains with some cell signaling and metabolism common to both: 1)

184 angiogenesis and vascular patterning and 2) inflammation. Five pathways, emerging from

185 angiogenesis and vascular patterning, were shared between HFrEF and HFpEF across AA,

186 EA, and HA women, namely, extracellular matrix (ECM)-receptor interaction, cell adhesion

187 molecules (CAMs), axon guidance, netrin-1 signaling, and developmental biology (Figure

188 1). The five shared pathways were highly interconnected as demonstrated by a shared

189 common set of 256 genes among them (Figure 2).

190 In addition, we found six pathways specifically enriched for HFpEF across AA and

191 EA, namely, adherens junction, endocytosis, phosphatidylinositol signal system, vascular

192 smooth muscle contraction, and heparan sulfate/heparin (HS)-glycosaminoglycan (GAG)

193 biosynthesis and degradation; all of which corresponded to the domain of angiogenesis and

194 vascular patterning (Figures 1 and 3). Of the aforementioned six HFpEF-specific pathways,

195 the first three pathways were further replicated in the WHI-HA participants. 
197 Identification of Key Drivers for HFpEF and HFrEF

198 In the KD analysis to identify potential genes that played a central role in the

199 significant pathways for HF, we used eight different regulatory or interaction networks that

200 capture gene-gene or protein-protein interactions among the pathways. The top $10 \mathrm{KD}$ genes

201 for the five shared pathways (developmental biology, axon guidance, netrin-1 signaling,

202 ECM-receptor interaction, and CAMs) between HFrEF and HFpEF across two ethnicities are

203 COL1A1, COL1A2, COL3A1, COLAA2, COL5A1, and COL6A3 from ECM and axon

204 guidance pathways, and HLA-DQA1, HLA-DQB1, HLA-DRB1, and HLA-DMB from CAMs

205 pathway (Figure 2 and Supplemental Figure 2). For the six pathways specific for HFpEF,

206 the top $10 \mathrm{KD}$ genes are MYH11, MYLK and PRKACB from vascular smooth muscle

207 contraction, $P R K C G, P I K 3 R 1$ from phosphatidylinositol signal system, $H G S, E G F$, and

208 SH3KBP1 from endocytosis, and CTNNBI and RACl from adherens junction (Figure 3 and

209 Supplemental Figure 2). Variants in the identified top KD genes collectively account for 15-

$21019 \%$ and $15-16 \%$ variations of HFrEF and HFpEF among women in the WHI.

\section{Discussion}

213 In this GWAS analysis of 7,982 AA and 4,133 EA women from the WHI, we

214 validated one previously reported genetic locus and allocated it to HFpEF, and additionally

215 discovered one HFrEF and five HFpEF novel genetic loci of potential importance. Also, five

216 biological pathways appeared to be shared for both HFrEF and HFpEF across AA, EA, and

217 HA women, and six pathways were specific for HFpEF across AA and EA women. Our

218 results suggested the presence of core mechanisms across HF subtypes (HFrEF and HFpEF),

219 such as vascular remodeling and inflammation alone with some common overlapping

220 mechanisms of cell signaling and metabolism. It is important to note the paucity of

221 cardiomyocyte-specific gene variants, including those for nuclear envelope proteins, 
222 sarcomere proteins, cytoskeletal, and calcium regulatory proteins, given their extensive

223 involvement in familial dilated cardiomyopathies. Our data highlight the genetic and/or

224 biological significance of the vascular remodeling and inflammation, rather than that of the

225 cardiomyocyte in the acquisition of HFpEF and HFrEF.

226 Given the increased diversity of gene involvement, the genetic architecture underlying

227 HFrEF and HFpEF remains challenging to delineate. We validated one previously reported

228 locus close to $A P O E$ and $A P O C$, and further allocated it to HFpEF. Genes in the

229 apolipoprotein family ( $A P O E, A P O C 1, A P O C 2$, etc.) encode lipid transport proteins that

230 regulate cholesterol metabolism and are associated with obesity and cardiovascular

231 disease ${ }^{27,28}$. Of note, we were not able to validate other suggestive HF loci reported from

232 previous European-based cohorts, which may be due to effect modifications by sex and/or

233 ethnicity.

234 In addition, we discovered one HFrEF and five HFpEF loci from intergenic regions.

235 Variant rs12719020, associated with HFrEF, is located upstream $(<20 \mathrm{~Kb})$ to $C O B L$, a gene

236 related to vasculitis and type 1 diabetes $^{29}$. For variants associated with HFpEF, rs 12067046 is

237 located $500 \mathrm{~Kb}$ downstream of PLXNA2, which is related to the development of blood

$238 \operatorname{vessel}^{30}$ and inflammatory-induced immune disorders ${ }^{31}$; the linkage disequilibrium (LD)

239 block around rs 12599260 is upstream $(5 \mathrm{~Kb})$ to HEATR3, which regulates inflammatory

240 immune response ${ }^{32}$; rs149663839 is located upstream (50 Kb) to $C A T$, a key antioxidant

241 enzyme, which is hypothesized to play a role in the development of many chronic or late-

242 onset diseases such as $\mathrm{HF}^{33}$; ACTAl $(60 \mathrm{~Kb}$ to the LD block around rs114553497) and

243 CALD1 (5 Kb to rs 10229703) are fundamental genes for skeletal/smooth muscle contraction

244 and had been linked to pulmonary hypertension in animal studies ${ }^{34,35}$ (Table 2 and

245 Supplemental Table 1). 
246 Genetic pathway and network analysis, as novel approaches to integrate genetic

247 signals that complements current GWAS analysis, have been yielded new insight into the

248 biology of coronary heart disease ${ }^{36}$, type 2 diabetes $^{25}$, obesity ${ }^{37}$, and LV function ${ }^{38}$. Our

249 pathway-based analysis revealed five consistent pathways between HFrEF and HFpEF across

250 the two ethnicities (Table 3). All the five pathways were linked to angiogenesis and vascular

251 patterning, among which three pathways, ECM-receptor interaction, CAMs, and Netrin-1

252 signaling were also linked to inflammation (Figure 1). From the five pathways shared by

253 both HFrEF and HFpEF, three pathways, axon guidance, ECM-receptor interaction, and

254 CAMs, had been implicated previously in thromboembolic cardiovascular disease, type 2

255 diabetes, and LV function ${ }^{25,38}$.

256 There appears to be a substantial overlap in the major areas of inflammation and

257 angiogenesis among shared pathways between HFrEF and HFpEF. The ECM is an intricate

258 network composed of multidomain macromolecules organized to support mechanical and

259 structural properties of cells and tissue but also to control behavioral characteristics of cells,

260 including proliferation, adhesion, migration, polarity and differentiation ${ }^{39,40}$. Major

261 components include collagens, proteoglycans, elastin, and cell-binding glycoproteins, each

262 with distinct physical and biochemical properties. ECM molecules connect to the cells

263 through integrins, syndecans, and other receptors which provides signaling input in addition

264 to mechanical support ${ }^{41}$. This ECM-receptor interaction contributes to angiogenesis and

265 vascular patterning in multiple ways, including the organization and maintenance of gradients

266 for angiogenic factors like vascular endothelial growth factor (VEGF)- $\mathrm{A}^{42,43}$. Endothelial

267 ECM receptors like intergrins play a critical role in adhesion and migration via control of

268 cytoskeletal dynamics while at the same time directing cell-cell interactions through

269 pathways like Notch signaling in order to coordinate sprouting and tube organization in early

270 capillary networks ${ }^{44}$. ECM-CAM interactions also have the ability to influence inflammatory 
271 state of both vascular and immune cells through focal adhesion complexes comprised of

272 integrins, protein kinases such as focal adhesion kinase (FAK), Src and many other kinases,

273 adaptor proteins such as Shc, signaling intermediates such as phosphoinositide 3-kinase

274 (PI3K), Rho and Rac GTPases, and actin binding cytoskeletal proteins ${ }^{45}$. Further, cardiac

275 ECM (primarily collagen I) may also play a critical role in providing a platform for

276 cardiomyocytes to maintain structure and function, and any change in ECM properties

277 following an insult has potential to drive the progression toward HF, including myocardial

278 fibrosis and altered ECM protein orientation ${ }^{46,47}$. Details regarding the main functions of

279 other identified pathways can be found in Supplemental Table 4.

280 Taken together, the above identified pathways highlight the previously known but

281 likely underappreciated importance of angiogenesis and vascular patterning as well as

282 inflammation on HF that appear to link HFrEF and HFpEF to other cardio-metabolic health

283 outcomes via multiple mechanisms. The fact that these pathways were consistently identified

284 across multiple ethnicities further highlights a convergent or central role in the joint

285 mechanisms between interrelated cardiac diseases.

286 We additionally identified six pathways specifically for HFpEF across ethnicities. All

287 the six pathways were linked to angiogenesis and vascular patterning, from which three

288 pathways (phosphatidylinositol signal system, HS-GAG biosynthesis and degradation) were

289 additionally linked to inflammation (Figure 1). None of the six HFpEF pathways have

290 previously been implicated in pathway-based studies, making these findings novel.

291 Specifically, the phosphatidylinositol signaling system is critically linked to

292 inflammation and vascular remodeling, and in particular angiogenesis and vascular

293 patterning. Activation of the PI3K pathway can occur in response to a variety of extracellular

294 (e.g., ECM, CAM) as well as growth factor (e.g., fibroblast growth factor, VEGF-A)

295 signaling and can regulate broad spectrum of molecular functions which involve 
296 proliferation, adhesion, migration, invasion, metabolism and cell survival ${ }^{48,49}$. Activation of

297 the PI3K pathway involves recruitment via Src-homology 2 (SH2) to phosphotyrosine

298 residues on the intracellular portion of membrane receptors, followed by phosphorylation of

299 phosphatidylinositol-4,5-bisphosphate (PIP2) to generate the second messenger molecule

300 PIP3. The Akt family of serine/threonine kinases has been shown to be the primary

301 downstream mediator of the effects of PI3K. Through the phosphorylation of IKB kinase

302 (IKK) and activation of nuclear factor $\kappa \mathrm{B}(\mathrm{NF}-\kappa \mathrm{B})$ transcriptional activity, Akt leads to

303 upregulation of inflammatory and prosurvival genes ${ }^{50-53}$. Akt can also activate mTOR,

304 resulting in stabilization of hypoxia inducible factor-1 (HIF-1) and consequent expression of

305 VEGF-A in order to promote angiogenesis ${ }^{54-56}$. PI3K signaling may also play a role in

306 regulating cardiomyocyte size, survival, and inflammation during cardiac hypertrophy and

$307 \mathrm{HF}$, in part via calcium signaling ${ }^{57,58}$. Details regarding the main functions of other identified

308 pathways can be found in Supplemental Table 4. Detailed knowledge of these relationships

309 at the molecular level will allow researcher to understand the distinct mechanisms underlying

310 HFpEF and enable the development of effective therapeutic strategies.

311 In addition, we observed two pathways with highly significant $P$ values: Rac1

312 pathway for HFrEF within EA and HFpEF within AA, and signaling by bone morphogenetic

313 proteins (BMP) pathway for HFpEF among AA (Supplemental Tables 2, 3, and 4). Rac1 is

314 a GTPase protein, a member of the Rac subfamily of the Rho family of GTPases, and plays a

315 critical role in inflammation and vascular remodeling. Activated Rac1 can promote NF- $\mathrm{BB}$

316 signaling and reactive oxygen species (ROS) production via nicotinamide adenine

317 dinucleotide phosphate (NADPH) oxidase, both known activators of the NACHT, LRR, and

318 PYD domains-containing protein 3 (NLRP3) inflammasome protein complex that promotes

319 expression of the critical inflammatory cytokine, interleukin-1 $\beta$ (IL-1 $\beta)^{59-61}$. A number of

320 studies have found that activation of Rac1 is associated with atrial fibrillation ${ }^{62}$, 
321 atherosclerotic calcification ${ }^{63}$, cardiac hypertrophy ${ }^{64}$, and $\mathrm{HF}^{65}$, suggesting Rac1 may have

322 strong potential as a new therapeutic target ${ }^{66}$. BMPs belong to transforming growth factor

323 beta (TGF $\beta$ ) superfamily, which is one of the most potent profibrogenic cytokine systems

324 governing cardiac fibrosis ${ }^{67}$. BMP signaling is also increasingly recognized for its influence

325 on endocrine-like functions in postnatal cardiovascular and metabolic homeostasis ${ }^{68}$. Some

326 BMP molecules, such as BMP9 and BMP10, had been found to reduce pulmonary arterial

327 hypertension, cardiac fibrosis, and myocardial infarction, thereby providing potentially

328 benefits for HF patients ${ }^{68,69}$.

329 The KD gene analysis prioritized KD genes of coronary heart disease ${ }^{70}$, type 2

330 diabetes $^{25}$, and obesity ${ }^{71}$, but has not been performed for HF. In our KD gene analysis based

331 on shared pathways between $\mathrm{HFrEF}$ and HFpEF, we found that the KD genes belong to the

332 collagen gene family, shared between axon guidance and ECM-receptor interaction, and

333 HLA genes from CAMs pathway. HLA gene family members are components of the major

334 histocompatibility complex (MHC) and play a central role in the immune system with

335 established allelic contributions to type 1 diabetes susceptibility ${ }^{72}$, and a host of inflammatory

336 disorders, including rheumatoid arthritis ${ }^{73}$, Sjögren's ${ }^{74}$, ulcerative colitis ${ }^{75}$, and systemic

337 lupus erythematosus ${ }^{76}$. Collagen gene family, as previously described, encodes proteins to

338 regulate vascular patterning and maintain the structure and function of cardiomyocytes.

339 These KD genes further highlight the effect of angiogenesis, vascular patterning and

340 inflammation in HF. Importantly, genes COL1A1 and COL3A1 were also found to be the KD

341 genes for thromboembolic cardiovascular disease and type 2 diabetes $^{25}$; thus, showing

342 potential shared biological mechanisms underlying these interrelated diseases as well as the

343 pleiotropic effects of these KD genes. COL $4 A 2$ is a critical component of the basement

344 membrane, and loss of function leads to disordered capillary networks during

345 angiogenesis ${ }^{77,78}$. The C-terminal portion of COLAA2 is a potent inhibitor of angiogenesis, 
346 prevents proliferation and migration of endothelial cells and induces apoptosis ${ }^{79}$. Moreover,

347 variants of COLAA2 are implicated in vascular cell survival, atherosclerotic plaque stability

348 and risk of myocardial infarction, as well as hemorrhagic stroke $e^{80,81}$.

349 The KD genes for HFpEF were mainly from the vascular smooth muscle contraction,

350 phosphatidylinositol signal system and endocytosis pathways, which further highlight the

351 functional roles of inflammation and systemic vascular remodeling in the pathogenesis of

352 HFpEF. Two examples of genes intricately linked to vascular wall mechanics rather than

353 cardiomyocyte mechanics include MYH11 and MYLK. MYH11 encodes one of the smooth

354 muscle cell myosin heavy chains, and variants are associated with familial thoracic aneurysm

355 syndrome ${ }^{82,83}$. MYLK encodes a myosin light chain kinase that is implicated in inflammatory

356 responses, apoptosis, and vascular permeability. Variants of $M Y L K$ are associated with

357 arterial and aortic aneurysmal disease ${ }^{84,85}$.

358 Several strengths and limitations need to be considered when interpreting these

359 findings. First, this study represents the first attempt to systematically examine and integrate

360 genetic variants for HF phenotypic subtypes using pathway and network approaches with

361 special emphasis on revealing mechanistic similarities and differences between HFrEF and

362 HFpEF with higher statistical efficiency. The second strength is the large, previously

363 validated and high-quality phenotyping of women of different ethnic backgrounds, which

364 allows the detection of HF mechanisms shared across ethnicities. Thirdly, two additional

365 high-quality cohorts, JHS and FHS, served as replication populations supporting the

366 robustness of our findings. One major limitation is that our results were based upon germline

367 mutations. Therefore, it is unclear whether mutations in the identified genomic regions and

368 pathways would impact downstream expression levels in particular tissues of interest, and

369 whether the identified genes and pathways are up-/down-regulated before and after HF

370 events. This highlights the critical need for future studies that will quantify the downstream 
371 gene expression changes by comparing population with and without HF. Moreover, it will be

372 important to validate these results in men in order to examine the effect of sex on HF and to

373 replicate our finding using suitable animal models of HF in order to further validate these

374 newly discovered pathways.

375

\section{Conclusion}

This study validated previously identified locus and defined novel loci for HF and its

378 subtypes, implicating specific molecular pathways, some shared and others unique, that

379 contribute to HF and its subtypes. We highlight the significant mechanistic role of the

380 inflammation and vascular remodeling (angiogenesis and vessel patterning) in the genetic

381 signals associated with HFpEF and HFrEF, supporting the concept that HF is largely a

382 disease of the systemic vasculature. Finally, this work defines several leading and novel targets and pathways for risk stratification and design of potential new HF interventions. 


\section{Funding}

386 This study is funded by the American Heart Association grant 17UNPG33750001.

388 Acknowledgements

389 The WHI program is funded by the National Heart, Lung, and Blood Institute, National

390 Institutes of Health, U.S. Department of Health and Human Services through contracts

391 HHSN268201600018C, HHSN268201600001C, HHSN268201600002C,

392 HHSN268201600003C, and HHSN268201600004C.

393

394 The Jackson Heart Study (JHS) is supported and conducted in collaboration with Jackson

395 State University (HHSN268201800013I), Tougaloo College (HHSN268201800014I), the

396 Mississippi State Department of Health (HHSN268201800015I) and the University of

397 Mississippi Medical Center (HHSN268201800010I, HHSN268201800011I and

398 HHSN268201800012I) contracts from the National Heart, Lung, and Blood Institute

399 (NHLBI) and the National Institute on Minority Health and Health Disparities (NIMHD).

400 The authors also wish to thank the staffs and participants of the JHS.

401

402 From the Framingham Heart Study of the National Heart Lung and Blood Institute of the

403 National Institutes of Health and Boston University School of Medicine. This project has

404 been funded in whole or in part with Federal funds from the National Heart, Lung, and Blood

405 Institute, National Institutes of Health, Department of Health and Human Services, under

406 Contract No. 75N92019D00031.

407

408 Dr. Alan R. Morrison is supported by the Research Project Grant NIH NHLBI

409 R01HL139795, the Institutional Development Award (IDeA) from NIH NIGMS 
410 P20GM103652, and the Career Development Award Number 7IK2BX002527 from the

411 United States Department of Veterans Affairs Biomedical Laboratory Research and

412 Development Program.

413

\section{Disclaimer}

415 The views expressed in this manuscript are those of the authors and do not necessarily

416 represent the views of the National Heart, Lung, and Blood Institute; the National Institutes

417 of Health, the Department of the Veterans Affairs; or the U.S. Department of Health and

418 Human Services. 


\section{Tables and Figures}

Table 1. Baseline Characteristics of African and European American Women in Study Populations ${ }^{\text {a }}$

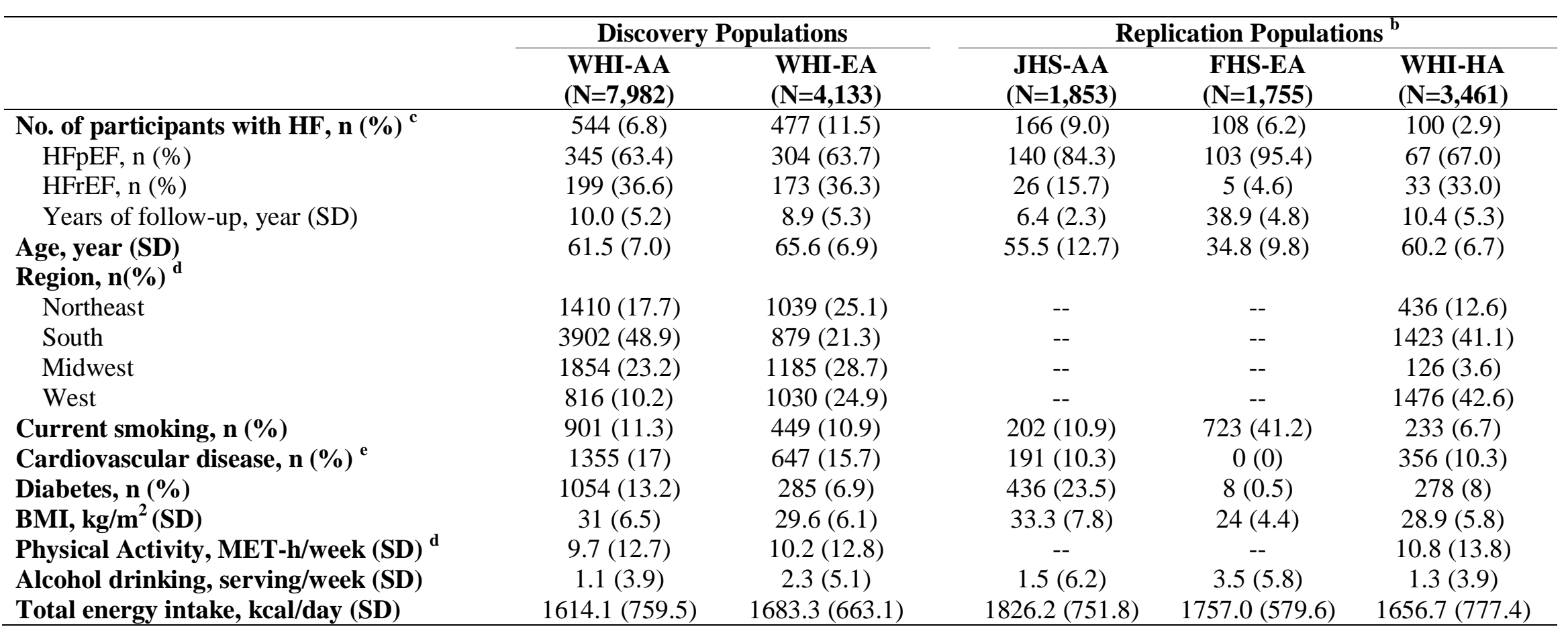

Abbreviations: AA (African American), ADHF (acute decompensated heart failure), BMI (body mass index), EA (European American), FHS (Framingham Heart Study), HA (Hispanic American), HF (heart failure), HFpEF (heart failure with preserved ejection fraction), HFrEF (heart failure with reduced ejection fraction), JHS (Jackson Heart Study), LVEF (left ventricular ejection fraction), SD (standard deviation), WHI (Women's Health Initiative Study). 
${ }^{a}$ Continuous variables were presented as mean (SD).

${ }^{\mathrm{b}} 62.0 \%$ in the JHS and $52.5 \%$ in the FHS participants were women.

${ }^{\mathrm{c}} \mathrm{HF}$ was defined as having ADHF or chronic stable HF, and further classified as HFrEF (LVEF< 45\%) or HFpEF (LVEF $\geq 45 \%$ ). For WHI patients with ADHF, LVEF closest to the diagnosis date of ADHF was used, and for patients with chronic stable HF, baseline LVEF or lowest estimated LVEF on medical records were used to classify HF subtypes. In the JHS, considering over 50\% of ADHF patients were missing LVEF, baseline LVEF was used to determine HFrEF and HFpEF for those without coronary heart disease before HF, and for chronic stable HF patients, baseline LVEF was used. In the FHS, HF was defined as incident or prevalent congestive HF. Since LVEF was not measured at baseline, LVEF closest to the diagnosis date of HF was used to define HFrEF and HFpEF. Participants without LVEF measurement were excluded in the analysis.

${ }^{\mathrm{d}}$ Results in the replication populations were not presented for variables which were not applicable (region) or measured in different scales (physical activity).

${ }^{\mathbf{e}}$ Cardiovascular disease was defined as self-reported coronary heart disease, heart failure, stroke, and peripheral artery disease at baseline. 
Table 2. Newly Discovered Loci for Heart Failure among Women across Ethnicities in the Women's Health Initiative Study, Jackson

Heart Study and Framingham Heart Study.

\begin{tabular}{|c|c|c|c|c|c|c|c|c|c|}
\hline \multirow{2}{*}{ Lead SNP } & \multirow{2}{*}{$\begin{array}{c}\text { Position } \\
\text { (hg19) }\end{array}$} & \multirow{2}{*}{ Candidate Gene $^{a}$} & \multirow{2}{*}{$\begin{array}{l}\text { Al 1/ } \\
\text { Al } 2\end{array}$} & \multicolumn{2}{|c|}{ Discovery $^{\text {b }}$} & \multicolumn{2}{|c|}{ Validation $^{\mathrm{c}}$} & \multicolumn{2}{|c|}{ Cross-ethnicity Meta-analysis ${ }^{\mathrm{d}}$} \\
\hline & & & & $\beta$ (SE) & $P$ value & $\beta$ (SE) & $P$ value & Direction & FDR-q value \\
\hline \multicolumn{10}{|c|}{ European American - HFrEF } \\
\hline rs 12719020 & $7: 51066547$ & Near $C O B L$ & $\mathrm{~A} / \mathrm{C}$ & $1.08(0.2)$ & $3.1 \times 10^{-6}$ & $2.59(0.9)$ & 0.02 & $+++?+{ }^{\mathrm{e}}$ & $8.8 \times 10^{-4}$ \\
\hline \multicolumn{10}{|c|}{ European American - HFpEF } \\
\hline rs12599260 & $16: 50093238$ & Near HEATR3 & $\mathrm{A} / \mathrm{G}$ & $-0.42(0.1)$ & $4.9 \times 10^{-6}$ & $-0.43(0.2)$ & $5.5 \times 10^{-3}$ & +---- & 0.01 \\
\hline \multicolumn{10}{|c|}{ African American - HFpEF } \\
\hline rs12067046 & $1: 208913216$ & Near PLXNA2 & $\mathrm{A} / \mathrm{G}$ & $0.47(0.1)$ & $4.2 \times 10^{-6}$ & $0.44(0.2)$ & $6.6 \times 10^{-3}$ & ++-++ & $7.2 \times 10^{-4}$ \\
\hline rs114553497 & $1: 229507916$ & Near CCSAP, ACTAI & $\mathrm{T} / \mathrm{C}$ & $0.92(0.2)$ & $5.2 \times 10^{-7}$ & $0.67(0.3)$ & 0.04 & $+?-+?$ & $1.6 \times 10^{-4}$ \\
\hline rs10229703 & $7: 134661230$ & Near $C A L D 1, A G B L 3$ & $\mathrm{~A} / \mathrm{G}$ & $0.49(0.1)$ & $3.6 \times 10^{-6}$ & $0.41(0.2)$ & 0.02 & +--+- & $7.6 \times 10^{-3}$ \\
\hline rs149663839 & $11: 34408645$ & Near $A B T B 2, C A T$ & $\mathrm{~A} / \mathrm{G}$ & $2.61(0.9)$ & $1.4 \times 10^{-6}$ & $1.69(0.8)$ & $5.1 \times 10^{-3}$ & $+? ?+?$ & $7.1 \times 10^{-6}$ \\
\hline
\end{tabular}

Abbreviations: Al (allele), FDR (false discovery rate), HFpEF (heart failure with preserved ejection fraction), HFrEF (heart failure with reduced ejection fraction), SE (standard error), SNP (single-nucleotide polymorphism).

${ }^{\text {a }}$ Closest genes within $\pm 300 \mathrm{~kb}$ of the lead SNPs.

${ }^{\mathrm{b}}$ The discovery populations were African Americans $(\mathrm{N}=7,982)$ and European Americans $(\mathrm{N}=4,133)$ within the Women's Health Initiative study.

${ }^{\mathrm{c}}$ The validation populations were African Americans women $(\mathrm{N}=1,853)$ in the Jackson Heart Study and European American women $(\mathrm{N}=1,755)$ in the Framingham Heart Study.

d The cross-ethnicity meta-analysis was performed among African Americans, European Americans, and Hispanic Americans in the Women's health Initiative study, and the Jackson Heart Study and Framing Heart Study.

e The following symbols denote the directions of the ethnicity-specific GWAS results: + denotes a positive association between the genetic variant and HF; - denotes a negative association between the genetic variant and HF; and ? denotes the tested variant was not available in the population. 
Table 3. Biological Pathways Enriched for HFrEF and HFpEF among African and European American Women across Ethnicities ${ }^{\text {a }}$

\begin{tabular}{|c|c|c|c|c|c|c|}
\hline \multirow{2}{*}{ Pathway } & \multicolumn{3}{|c|}{ HFrEF } & \multicolumn{3}{|c|}{ HFpEF } \\
\hline & EA & $\overline{\mathbf{A A}}$ & HA & EA & $\mathbf{A A}$ & HA \\
\hline \multicolumn{7}{|l|}{ Angiogenesis and Vascular Patterning } \\
\hline Extracellular matrix-receptor interaction & $X$ & $\mathrm{X}$ & $X$ & $X$ & $\mathrm{X}$ & $X$ \\
\hline Cell adhesion molecules & $\mathrm{X}$ & $\mathrm{X}$ & $\mathrm{X}$ & $X$ & $\mathrm{X}$ & $\mathrm{X}$ \\
\hline Axon guidance & $\mathrm{X}$ & $\mathrm{X}$ & $\mathrm{X}$ & $\mathrm{X}$ & $\mathrm{X}$ & $\mathrm{X}$ \\
\hline Netrin-1 signaling & $X$ & $\mathrm{X}$ & $\mathrm{X}$ & $X$ & $\mathrm{X}$ & $\mathrm{X}$ \\
\hline Developmental biology & $X$ & $\mathrm{X}$ & $X$ & $X$ & $\mathrm{X}$ & $\mathrm{X}$ \\
\hline Adherens junction & & $\mathrm{X}$ & $\mathrm{X}$ & $X$ & $\mathrm{X}$ & $\mathrm{X}$ \\
\hline Endocytosis & & $\mathrm{X}$ & $X$ & $X$ & $X$ & $\mathrm{X}$ \\
\hline Phosphatidylinositol signal system & & & & $X$ & $\mathrm{X}$ & $\mathrm{X}$ \\
\hline Vascular smooth muscle contraction & $\mathrm{X}$ & & $X$ & $X$ & $\mathrm{X}$ & \\
\hline HS-GAG biosynthesis & & $\mathrm{X}$ & & $\mathrm{X}$ & $\mathrm{X}$ & \\
\hline HS-GAG degradation & & & & $X$ & $\mathrm{X}$ & \\
\hline Mucin type O-glycan biosynthesis & & $\mathrm{X}$ & $X$ & $X$ & & $\mathrm{X}$ \\
\hline Pre-Notch expression and processing & & & & $X$ & & $\mathrm{X}$ \\
\hline Signaling by BMP & & & & & $\mathrm{X}$ & \\
\hline Cell-cell junction organization & & & $X$ & & $\mathrm{X}$ & \\
\hline Intrinsic pathway & $X$ & & $\mathrm{X}$ & & & $\mathrm{X}$ \\
\hline \multicolumn{7}{|l|}{ Inflammation } \\
\hline Extracellular matrix-receptor interaction & $\mathrm{X}$ & $\mathrm{X}$ & $X$ & $X$ & $\mathrm{X}$ & $\mathrm{X}$ \\
\hline Cell adhesion molecules & $X$ & $\mathrm{X}$ & $X$ & $X$ & $\mathrm{X}$ & $\mathrm{X}$ \\
\hline Netrin-1 signaling & $\mathrm{X}$ & $\mathrm{X}$ & $X$ & $X$ & $\mathrm{X}$ & $\mathrm{X}$ \\
\hline Phosphatidylinositol signal system & & & & $X$ & $\mathrm{X}$ & $\mathrm{X}$ \\
\hline HS-GAG biosynthesis & & $\mathrm{X}$ & & $X$ & $\mathrm{X}$ & \\
\hline HS-GAG degradation & & & & $X$ & $\mathrm{X}$ & \\
\hline Rac1 pathway & $\mathrm{X}$ & & & & $\mathrm{X}$ & \\
\hline NRAGE signals death through JNK & & $\mathrm{X}$ & & $\mathrm{X}$ & & \\
\hline Signaling by Rho GTPases & & $\mathrm{X}$ & $\mathrm{X}$ & $X$ & & $\mathrm{X}$ \\
\hline Cell-cell junction organization & & & $X$ & & $\mathrm{X}$ & \\
\hline Intrinsic pathway & $\mathrm{X}$ & & $\mathrm{X}$ & & & $\mathrm{X}$ \\
\hline
\end{tabular}

Abbreviations: AA (African Americans), BMP (Bone morphogenetic proteins), EA (European Americans), GAG (glycosaminoglycan), HA (Hispanic American), HFpEF (heart failure with preserved ejection fraction), $\mathrm{HFrEF}$ (heart failure with reduced ejection fraction), HS (Heparan sulfate), JNK (JUN Kinase).

${ }^{a}$ Biological pathways presented in the table were those identified from AA and EA women in the Women's Health Initiative Study, and validated in the Jackson Heart Study and Framingham Heart Study. A pathway was marked with " $X$ " when nominal $P$ value from GSA-SNP or Mergeomics $<0.05$ in the Women's Health Initiative Study 


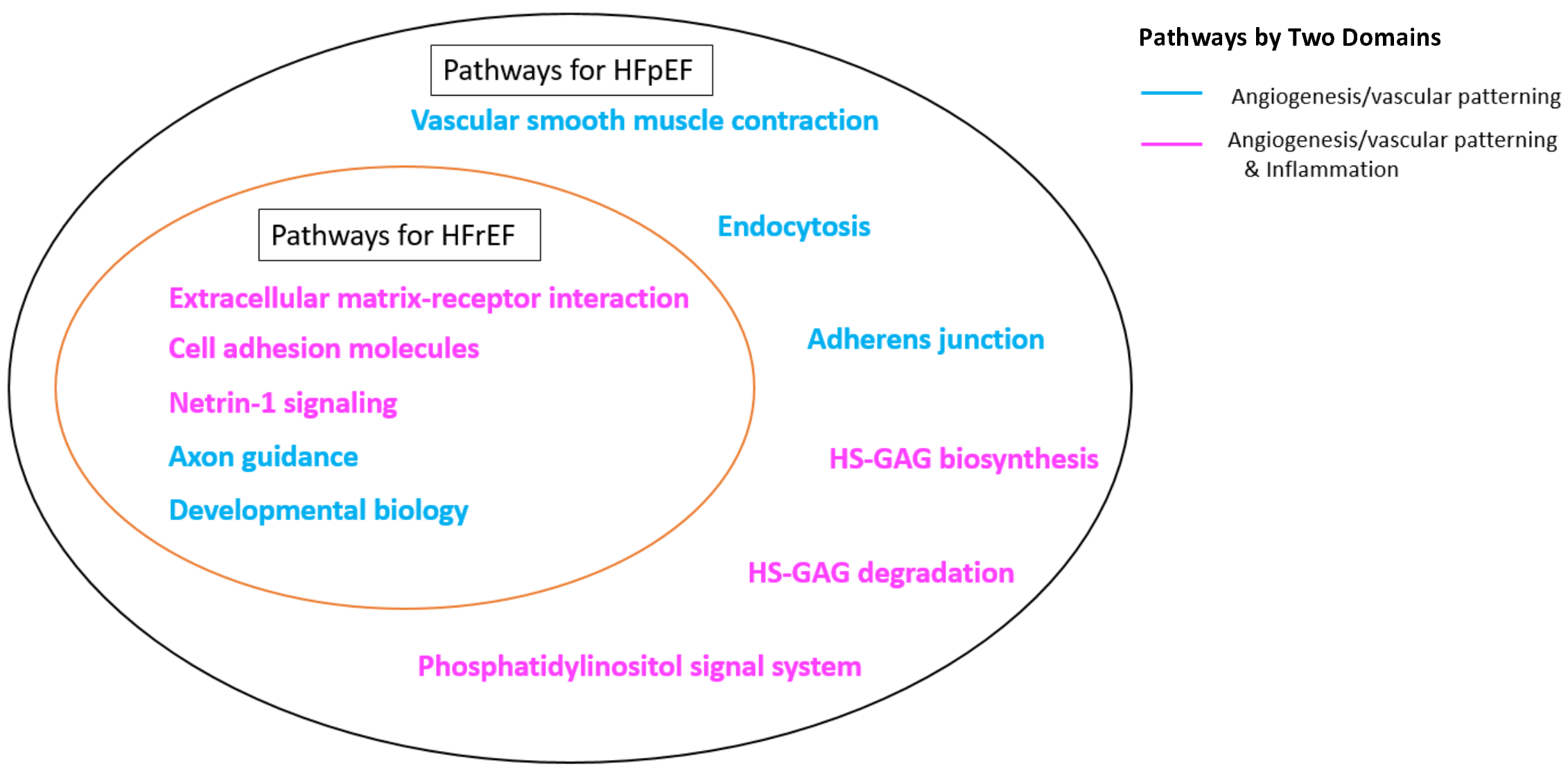

Figure 1. Venn Diagram for Biological Pathways Enriched for HFrEF and HFpEF among African and European American Women across Ethnicities 
Abbreviations: GAG (glycosaminoglycan), HFpEF (heart failure with preserved ejection fraction), HFrEF (heart failure with reduced ejection fraction), HS (Heparan sulfate). 


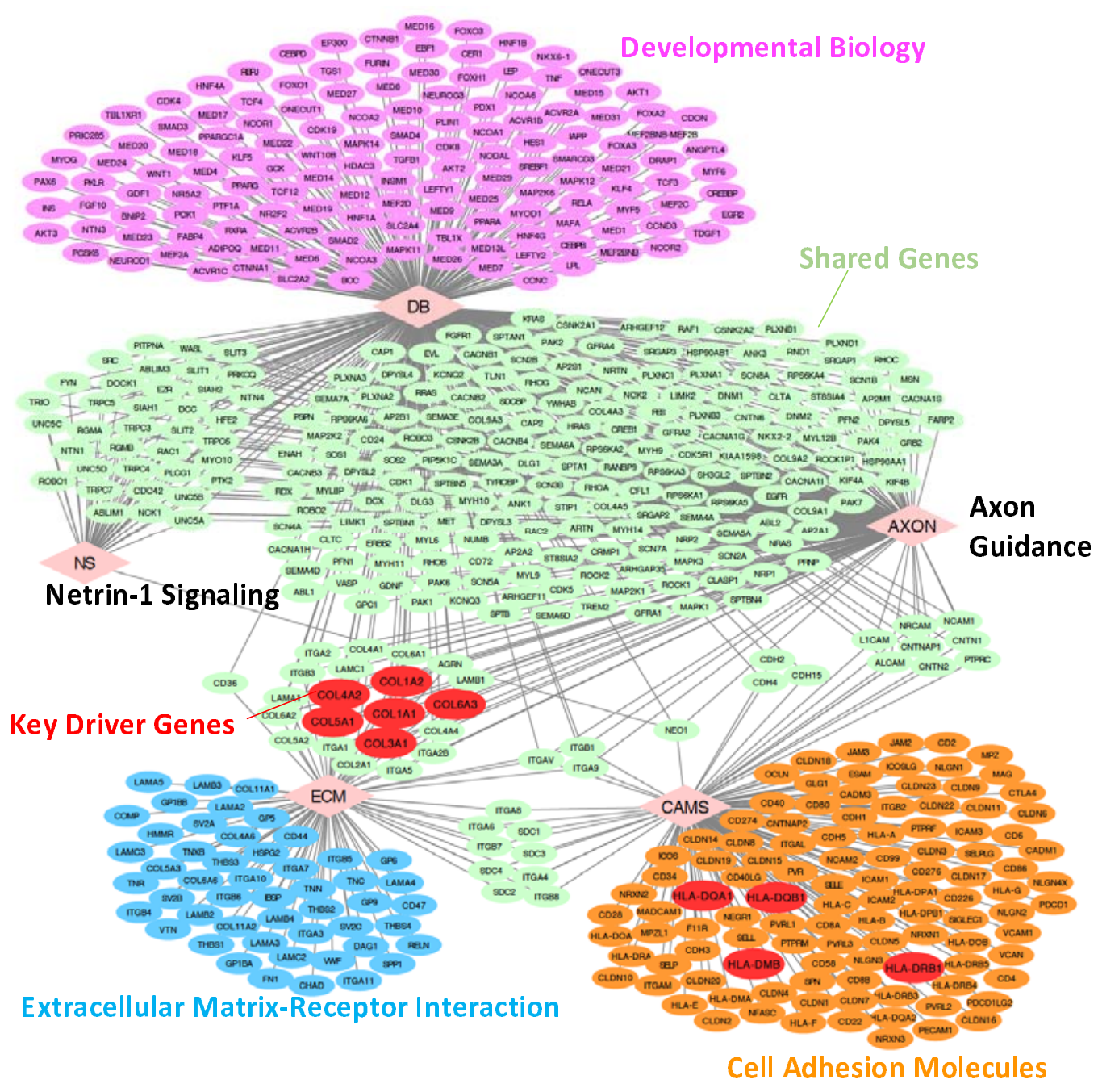

Figure 2. Network of 5 Pathways Enriched for HFrEF and HFpEF with Top 10 Key

Driver Genes among African and European American Women.

The diamond nodes represent pathway and the ellipse modes represent genes, and the edge shows the interaction, that is, the association between a gene and a pathway. The color nodes are: red, top 10 key driver genes; light green, genes involved in $\geq 2$ pathways; others are pathway-specific genes. The figure was created using Cytoscape ${ }^{86}$.

Abbreviations: AXON (axon guidance), CAMS (Cell adhesion molecules), DB (developmental biology), ECM (Extracellular matrix-receptor interaction), HFpEF (heart failure with preserved ejection fraction), $\mathrm{HFrEF}$ (heart failure with reduced ejection fraction), NS (Netrin-1 signaling). 


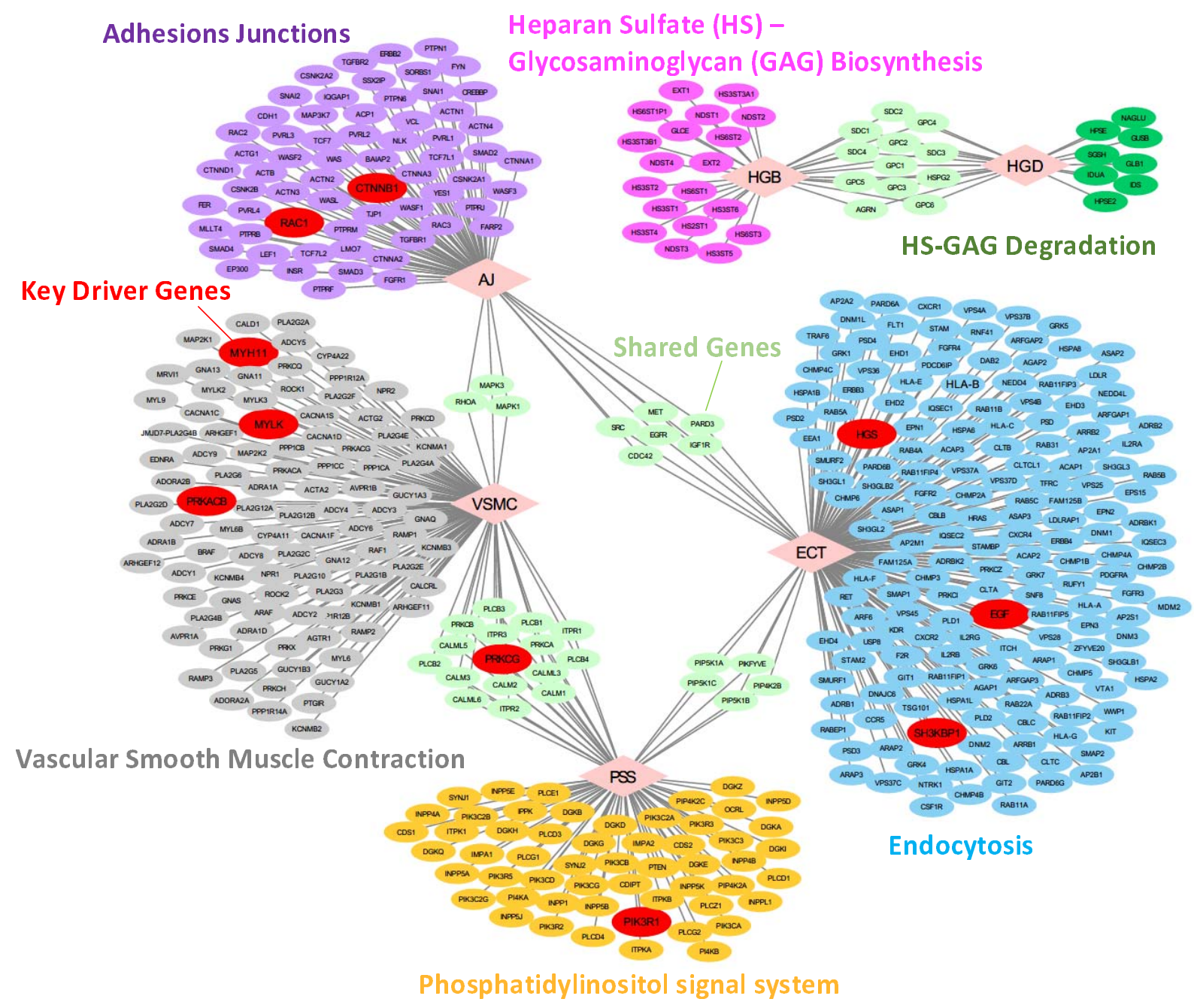

Figure 3. Network of 6 Pathways Enriched for HFpEF with Top 10 Key Driver Genes among African and European American Women.

The diamond nodes represent pathway and the ellipse modes represent genes, and the edge shows the interaction, that is, the association between a gene and a pathway. The color nodes are: red, top 10 key driver genes; light green, genes involved in $\geq 2$ pathways; others are pathway-specific genes. The figure was created using Cytoscape ${ }^{86}$. Abbreviations: AJ (adherens junction), ECT (endocytosis), HGB (heparan sulfateglycosaminoglycan biosynthesis), HGD (heparan sulfate-glycosaminoglycan degradation), HFpEF (heart failure with preserved ejection fraction), PSS (phosphatidylinositol signal system), VSMC (vascular smooth muscle contraction). 


\section{References}

1. Benjamin EJ, Virani SS, Callaway CW, et al. Heart Disease and Stroke Statistics2018 Update: A Report From the American Heart Association. Circulation 2018;137:e67e492.

2. $\quad$ Eaton CB, Pettinger M, Rossouw J, et al. Risk Factors for Incident Hospitalized Heart Failure With Preserved Versus Reduced Ejection Fraction in a Multiracial Cohort of Postmenopausal Women. Circulation Heart failure 2016;9.

3. Dries DL, Exner DV, Gersh BJ, Cooper HA, Carson PE, Domanski MJ. Racial differences in the outcome of left ventricular dysfunction. The New England journal of medicine 1999;340:609-16.

4. Bahrami H, Kronmal R, Bluemke DA, et al. Differences in the incidence of congestive heart failure by ethnicity: the multi-ethnic study of atherosclerosis. Archives of internal medicine 2008;168:2138-45.

5. Eaton CB, Abdulbaki AM, Margolis KL, et al. Racial and ethnic differences in incident hospitalized heart failure in postmenopausal women: the Women's Health Initiative. Circulation 2012;126:688-96.

6. Loehr LR, Rosamond WD, Chang PP, Folsom AR, Chambless LE. Heart failure incidence and survival (from the Atherosclerosis Risk in Communities study). The American journal of cardiology 2008;101:1016-22.

7. Yancy CW, Jessup M, Bozkurt B, et al. 2013 ACCF/AHA guideline for the management of heart failure: executive summary: a report of the American College of Cardiology Foundation/American Heart Association Task Force on practice guidelines. Circulation 2013;128:1810-52.

8. Schwartzenberg S, Redfield MM, From AM, Sorajja P, Nishimura RA, Borlaug BA. Effects of vasodilation in heart failure with preserved or reduced ejection fraction implications of distinct pathophysiologies on response to therapy. J Am Coll Cardiol 2012;59:442-51.

9. Zile MR, Brutsaert DL. New concepts in diastolic dysfunction and diastolic heart failure: Part II: causal mechanisms and treatment. Circulation 2002;105:1503-8.

10. Lopes LR, Elliott PM. Genetics of heart failure. Biochim Biophys Acta 2013;1832:2451-61.

11. Aragam KG, Chaffin M, Levinson RT, et al. Phenotypic Refinement of Heart Failure in a National Biobank Facilitates Genetic Discovery. Circulation 2018.

12. Franceschini N, Kopp JB, Barac A, et al. Association of APOL1 With Heart Failure With Preserved Ejection Fraction in Postmenopausal African American Women. JAMA Cardiol 2018;3:712-20.

13. Tayal U, Prasad S, Cook SA. Genetics and genomics of dilated cardiomyopathy and systolic heart failure. Genome Med 2017;9:20.

14. Nam D, Kim J, Kim SY, Kim S. GSA-SNP: a general approach for gene set analysis of polymorphisms. Nucleic acids research 2010;38:W749-54.

15. Arneson D, Bhattacharya A, Shu L, Makinen VP, Yang X. Mergeomics: a web server for identifying pathological pathways, networks, and key regulators via multidimensional data integration. BMC genomics 2016;17:722.

16. Design of the Women's Health Initiative clinical trial and observational study. The Women's Health Initiative Study Group. Controlled clinical trials 1998;19:61-109.

17. Rosamond WD, Chang PP, Baggett C, et al. Classification of heart failure in the atherosclerosis risk in communities (ARIC) study: a comparison of diagnostic criteria.

Circulation Heart failure 2012;5:152-9. 
18. Hall PS, Nah G, Howard BV, et al. Reproductive Factors and Incidence of Heart Failure Hospitalization in the Women's Health Initiative. Journal of the American College of Cardiology 2017;69:2517-26.

19. Willer CJ, Li Y Fau - Abecasis GR, Abecasis GR. METAL: fast and efficient metaanalysis of genomewide association scans.

20. Kyoto Encyclopedia of Genes and Genomes. 2018. (Accessed Oct 17th, 2018, at https://www.genome.jp/kegg/.)

21. Reactome. 2018. (Accessed Oct 17th, 2018, at https://reactome.org/.)

22. BioCarta. 2018. (Accessed Oct 17th, 2018, at

http://software.broadinstitute.org/gsea/msigdb/genesets.jsp?collection=CP:BIOCARTA.)

23. Zhu J, Zhang B, Smith EN, et al. Integrating large-scale functional genomic data to dissect the complexity of yeast regulatory networks. Nat Genet 2008;40:854-61.

24. Wang IM, Zhang B, Yang X, et al. Systems analysis of eleven rodent disease models reveals an inflammatome signature and key drivers. Mol Syst Biol 2012;8:594.

25. Chan KH, Huang YT, Meng Q, et al. Shared molecular pathways and gene networks for cardiovascular disease and type 2 diabetes mellitus in women across diverse ethnicities. Circulation Cardiovascular genetics 2014;7:911-9.

26. GWAS Catalog. 2018. (Accessed Oct 18th, 2018, at https://www.ebi.ac.uk/gwas/.)

27. Kei AA, Filippatos TD, Tsimihodimos V, Elisaf MS. A review of the role of apolipoprotein C-II in lipoprotein metabolism and cardiovascular disease. Metabolism 2012;61:906-21.

28. Haan MN, Mayeda ER. Apolipoprotein E Genotype and Cardiovascular Diseases in the Elderly. Curr Cardiovasc Risk Rep 2010;4:361-8.

29. Torn C, Hadley D, Lee HS, et al. Role of Type 1 Diabetes-Associated SNPs on Risk of Autoantibody Positivity in the TEDDY Study. Diabetes 2015;64:1818-29.

30. Sun Q, Liu S, Liu K, Jiao K. Role of Semaphorin Signaling During Cardiovascular Development. Journal of the American Heart Association 2018;7.

31. Takamatsu H, Kumanogoh A. Diverse roles for semaphorin-plexin signaling in the immune system. Trends Immunol 2012;33:127-35.

32. Zhang W, Hui KY, Gusev A, et al. Extended haplotype association study in Crohn's disease identifies a novel, Ashkenazi Jewish-specific missense mutation in the NF-kappaB pathway gene, HEATR3. Genes Immun 2013;14:310-6.

33. Ribeiro-Samora GA, Rabelo LA, Ferreira ACC, et al. Inflammation and oxidative stress in heart failure: effects of exercise intensity and duration. Braz J Med Biol Res 2017;50:e6393.

34. Wallner M, Eaton DM, Berretta RM, et al. A Feline HFpEF Model with Pulmonary Hypertension and Compromised Pulmonary Function. Sci Rep 2017;7:16587.

35. Moreno-Vinasco L, Garcia JG. Receptor tyrosine kinase inhibitors in rodent pulmonary hypertension. Adv Exp Med Biol 2010;661:419-34.

36. de las Fuentes L, Yang W, Davila-Roman VG, Gu C. Pathway-based genome-wide association analysis of coronary heart disease identifies biologically important gene sets. European journal of human genetics : EJHG 2012;20:1168-73.

37. Wang L, Jing J, Fu Q, et al. Association study of genetic variants at newly identified lipid gene TRIB1 with coronary heart disease in Chinese Han population. Lipids Health Dis 2015;14:46.

38. Wells QS, Veatch OJ, Fessel JP, et al. Genome-wide association and pathway analysis of left ventricular function after anthracycline exposure in adults. Pharmacogenet Genomics 2017;27:247-54. 
39. Lu P, Takai K Fau - Weaver VM, Weaver Vm Fau - Werb Z, Werb Z. Extracellular matrix degradation and remodeling in development and disease. LID -

10.1101/cshperspect.a005058 [doi] LID - a005058 [pii].

40. Mecham RP. Overview of extracellular matrix.

41. Gattazzo F, Urciuolo A, Bonaldo P. Extracellular matrix: a dynamic

microenvironment for stem cell niche.

42. Gerhardt H, Golding M Fau - Fruttiger M, Fruttiger M Fau - Ruhrberg C, et al. VEGF guides angiogenic sprouting utilizing endothelial tip cell filopodia.

43. Ruhrberg C, Gerhardt H Fau - Golding M, Golding M Fau - Watson R, et al. Spatially restricted patterning cues provided by heparin-binding VEGF-A control blood vessel branching morphogenesis.

44. Mettouchi A. The role of extracellular matrix in vascular branching morphogenesis. Cell Adh Migr 2012;6:528-34.

45. Mitra SK, Hanson Da Fau - Schlaepfer DD, Schlaepfer DD. Focal adhesion kinase: in command and control of cell motility.

46. Jourdan-Lesaux C, Zhang J, Lindsey ML. Extracellular matrix roles during cardiac repair. Life sciences 2010;87:391-400.

47. Bayomy AF, Bauer M, Qiu Y, Liao R. Regeneration in heart disease-Is ECM the key? Life sciences 2012;91:823-7.

48. Bader AG, Kang S Fau - Zhao L, Zhao L Fau - Vogt PK, Vogt PK. Oncogenic PI3K deregulates transcription and translation.

49. Dillon RL, White De Fau - Muller WJ, Muller WJ. The phosphatidyl inositol 3-kinase signaling network: implications for human breast cancer.

50. Kane LP, Shapiro Vs Fau - Stokoe D, Stokoe D Fau - Weiss A, Weiss A. Induction of NF-kappaB by the Akt/PKB kinase.

51. Ozes ON, Mayo LD, Gustin JA, Pfeffer SR, Pfeffer LM, Donner DB. NF- $\kappa B$

activation by tumour necrosis factor requires the Akt serine-threonine kinase. Nature 1999;401:82.

52. Romashkova JA, Makarov SS. NF-kappaB is a target of AKT in anti-apoptotic PDGF signalling.

53. Sizemore N, Leung S Fau - Stark GR, Stark GR. Activation of phosphatidylinositol 3kinase in response to interleukin-1 leads to phosphorylation and activation of the NF-kappaB p65/RelA subunit.

54. Li Y, Corradetti Mn Fau - Inoki K, Inoki K Fau - Guan K-L, Guan KL. TSC2: filling the GAP in the mTOR signaling pathway.

55. Tan C, Cruet-Hennequart S Fau - Troussard A, Troussard A Fau - Fazli L, et al. Regulation of tumor angiogenesis by integrin-linked kinase (ILK).

56. Zhong H, Chiles K, Feldser D, et al. Modulation of hypoxia-inducible factor $1 \alpha$ expression by the epidermal growth factor/phosphatidylinositol 3-kinase/PTEN/AKT/FRAP pathway in human prostate cancer cells: implications for tumor angiogenesis and therapeutics. Cancer research 2000;60:1541-5.

57. Ghigo A, Laffargue M, Li M, Hirsch E. PI3K and Calcium Signaling in Cardiovascular Disease. Circulation research 2017;121:282-92.

58. Aoyagi T, Matsui T. Phosphoinositide-3 kinase signaling in cardiac hypertrophy and heart failure. Current pharmaceutical design 2011;17:1818-24.

59. Hordijk PL. Regulation of NADPH oxidases: the role of Rac proteins. Circ Res 2006;98:453-62.

60. Arbibe L, Mira Jp Fau - Teusch N, Teusch N Fau - Kline L, et al. Toll-like receptor 2mediated NF-kappa B activation requires a Rac1-dependent pathway. 
61. Khan OM, Ibrahim Mx Fau - Jonsson I-M, Jonsson Im Fau - Karlsson C, et al. Geranylgeranyltransferase type I (GGTase-I) deficiency hyperactivates macrophages and induces erosive arthritis in mice.

62. Adam O, Frost G, Custodis F, et al. Role of Rac1 GTPase activation in atrial fibrillation. J Am Coll Cardiol 2007;50:359-67.

63. Ceneri N, Zhao L, Young BD, et al. Rac2 Modulates Atherosclerotic Calcification by Regulating Macrophage Interleukin-1beta Production. Arterioscler Thromb Vasc Biol 2017;37:328-40.

64. Satoh M, Ogita H, Takeshita K, Mukai Y, Kwiatkowski DJ, Liao JK. Requirement of Rac1 in the development of cardiac hypertrophy. Proc Natl Acad Sci U S A 2006;103:74327.

65. Do e Z, Fukumoto Y, Sugimura K, et al. Rho-kinase activation in patients with heart failure. Circ J 2013;77:2542-50.

66. Ridker PM, Everett BM, Thuren T, et al. Antiinflammatory Therapy with Canakinumab for Atherosclerotic Disease. The New England journal of medicine 2017;377:1119-31.

67. Leask A. TGFbeta, cardiac fibroblasts, and the fibrotic response. Cardiovasc Res 2007;74:207-12.

68. Morrell NW, Bloch DB, ten Dijke P, et al. Targeting BMP signalling in cardiovascular disease and anaemia. Nat Rev Cardiol 2016;13:106-20.

69. Morine KJ, Qiao X, York S, et al. Bone Morphogenetic Protein 9 Reduces Cardiac Fibrosis and Improves Cardiac Function in Heart Failure. Circulation 2018.

70. Makinen VP, Civelek M, Meng Q, et al. Integrative genomics reveals novel molecular pathways and gene networks for coronary artery disease. PLoS genetics 2014;10:e1004502.

71. Wang L, Perez J, Heard-Costa N, et al. Integrating genetic, transcriptional, and biological information provides insights into obesity. International journal of obesity (2005) 2018.

72. Roark CL, Anderson KM, Simon LJ, Schuyler RP, Aubrey MT, Freed BM. Multiple HLA epitopes contribute to type 1 diabetes susceptibility. Diabetes 2014;63:323-31.

73. Zanelli E, Breedveld Fc Fau - de Vries RR, de Vries RR. HLA association with autoimmune disease: a failure to protect?

74. Nakken B, Jonsson R Fau - Brokstad KA, Brokstad Ka Fau - Omholt K, et al. Associations of MHC class II alleles in Norwegian primary Sjogren's syndrome patients: implications for development of autoantibodies to the Ro52 autoantigen.

75. Garrity-Park MM, Loftus Ev Jr Fau - Sandborn WJ, Sandborn Wj Fau - Bryant SC, Bryant Sc Fau - Smyrk TC, Smyrk TC. MHC Class II alleles in ulcerative colitis-associated colorectal cancer.

76. Morris DL, Taylor Ke Fau - Fernando MMA, Fernando Mm Fau - Nititham J, et al. Unraveling multiple MHC gene associations with systemic lupus erythematosus: model choice indicates a role for HLA alleles and non-HLA genes in Europeans.

77. Poschl E, Schlotzer-Schrehardt U Fau - Brachvogel B, Brachvogel B Fau - Saito K, Saito K Fau - Ninomiya Y, Ninomiya Y Fau - Mayer U, Mayer U. Collagen IV is essential for basement membrane stability but dispensable for initiation of its assembly during early development.

78. Kuo DS, Labelle-Dumais C Fau - Gould DB, Gould DB. COL4A1 and COL4A2 mutations and disease: insights into pathogenic mechanisms and potential therapeutic targets.

79. Kamphaus GD, Colorado Pc Fau - Panka DJ, Panka Dj Fau - Hopfer H, et al. Canstatin, a novel matrix-derived inhibitor of angiogenesis and tumor growth. 
80. Meuwissen ME, Halley DJA-Ohoo, Smit LSA-Ohoo, et al. The expanding phenotype of COL4A1 and COL4A2 mutations: clinical data on 13 newly identified families and a review of the literature.

81. Yang W, Ng FL, Chan K, et al. Coronary-Heart-Disease-Associated Genetic Variant at the COL4A1/COL4A2 Locus Affects COL4A1/COL4A2 Expression, Vascular Cell Survival, Atherosclerotic Plaque Stability and Risk of Myocardial Infarction. PLoS Genet 2016;12:e1006127.

82. Zhu L, Vranckx R Fau - Khau Van Kien P, Khau Van Kien P Fau - Lalande A, et al. Mutations in myosin heavy chain 11 cause a syndrome associating thoracic aortic aneurysm/aortic dissection and patent ductus arteriosus.

83. Takeda N, Morita H, Fujita D, et al. A deleterious MYH11 mutation causing familial thoracic aortic dissection.

84. Wang L, Guo Dc Fau - Cao J, Cao J Fau - Gong L, et al. Mutations in myosin light chain kinase cause familial aortic dissections.

85. Lindsay ME, Dietz HC. The genetic basis of aortic aneurysm.

86. Shannon P, Markiel A, Ozier O, et al. Cytoscape: a software environment for integrated models of biomolecular interaction networks. Genome research 2003;13:2498-504. 